\title{
Observations of ionospheric flows and particle precipitation following a Sudden Commencement
}

\author{
E. Nielsen ${ }^{1}$, F. Honary ${ }^{2}$ \\ ${ }^{1}$ Max-Planck-Institute for Aeronomie, D-37191 Katlenburg-Lindau, FRG \\ ${ }^{2}$ Communications Research Centre, Faculty of Applied Sciences, Lancaster University, Lancaster, UK
}

Received: 18 October 1999 / Revised: 13 March 2000 / Accepted: 21 April 2000

\begin{abstract}
On May 4, 1998, at 0227 UT an interplanetary shock crossed the WIND spacecraft, and half an hour later a Sudden Commencement occurred. Coinciding with the Sudden Commencement a rapid intensification of the flux of particle precipitation into the ionosphere was observed. Evidence is presented that the ionospheric electric fields were influenced by the associated dynamic variations of the ionospheric conductivities. Following the initial phase the ionospheric flow speeds increased rapidly over the next $20 \mathrm{~min}$ to more than $2000 \mathrm{~m} / \mathrm{s}$, in agreement with an increased effective coupling of the solar wind energy to the magnetosphere following the interplanetary shock that caused the Sudden Commencement. These strong flows were meandering in latitude, a type of plasma flow modulation that has been reported before to occur during Omega band events: a string of alternating field-aligned currents propagating eastward. The riometer absorption was found to be at a minimum in regions associated with outward directed field aligned currents. The riometer absorption regions (the regions of particle precipitation) were drifting with $\mathbf{E} \times \mathbf{B}$ drift speed of the ionospheric electrons.
\end{abstract}

Key words: Interplanetary physics (interplanetary shocks) - Ionosphere (electric fields and currents) Magnetospheric physics (energetic particles, precipitating)

\section{Introduction}

Simultaneous observations of electron flows in the Eregion ionosphere, and of radio wave absorption caused by energetic particle precipitation into D-region, are presented during a time that encompasses the storm onset and the beginning of the main phase of a magnetic storm. It was an intense and dynamic event with an

Correspondence to: E. Nielsen initial phase (Akasofu, 1981) that lasted only a few minutes. About 20 min after onset the $D s t$ index started to decreased towards a minimum of $-216 \mathrm{nT}$, which it reached two and a half hours later. The purpose of the study is to use this intense event to examine the effects of an interplanetary shock colliding with the Earth's magnetosphere on particle precipitation and on magnetospheric/ionospheric convection during the early phases of the interaction.

It is well known that at the time of a Sudden Commencement a sudden burst of particle precipitation occurs that gives rise to a short-lasting intense increase in riometer absorption, known as Sudden Commencement Absorption (SCA; Ortner et al., 1962). This is also observed in the event presented. The rapid absorption variations are likely to be accompanied by rapid conductivity variations in the E-region. These data are used to examine the effects of rapid and dynamic Pedersen conductivity variations on the ionospheric electric field. The relation between conductivity and electric fields have been studied before for steady state conditions (Davies and Lester, 1999).

The riometer absorption variations following the SCA are characterised by recurrent spiky increases. These increases are associated with spatial regions of absorption, drifting with the ionospheric plasma in an $\mathbf{E} \times \mathbf{B}$ drift. Previous observations of the drift velocity of absorption patches have only demonstrated that the drift velocities were not inconsistent with an $\mathbf{E} \times \mathbf{B}$ drift (Hargreaves, 1970).

At the onset of a magnetic storm the solar wind pressure on the magnetosphere suddenly increases, thereby also increasing the energy input to the magnetosphere (Akasofu, 1981). If the interplanetary magnetic field is turning southward a further improvement in the energy coupling follows, either through steady reconnection (Dungey, 1961) or through patchy reconnection called flux transfer events Saunders et al. (1984). In response to the increased energy input the magnetospheric plasma flows to equalise variations in pressures in order to reach a new equilibrium. In this event is 
observed a rapid increase of the eastward (sunward) directed ionospheric electron flow velocities over $20 \mathrm{~min}$ following storm onset. The flows are meandering in latitude as if a series of spatial regions with alternating inward and outward pointing electric fields (or alternating inward and outward flowing field aligned currents) propagate in the eastward direction. This is a pattern known from Omega bands which are observed during the substorm recovery phase (Andre, 1982; Opgenoorth et al., 1983). Rather than considering such meandering flows to be specifically a substorm phenomena, it is suggested that meandering plasma flows results from irregular pulsations of the magnetosphere system as it is in transfer from one steady state to another. For this event the meandering flows serve to return the magnetosphere system to a new equilibrium corresponding to the conditions in interplanetary space following the Sudden Commencement. Thus, it is suggested that the meandering electron flows are triggered by the SC.

Following this phase a series of events occur, which are characterised by poleward turning flows, with the flows even turning westward at high latitudes. After several minutes, at the end of each event, the flow direction again returned to an eastward direction. These flow patterns indicate that the polar cap has expanded equatorward into the field-of-view of the ground-based experiments.

\section{Experiments}

The flow and absorption measurements were made with ground-based experiments that have large (partially overlapping) spatial fields-of-views. The experiments make continuous observations with good spatial and temporal resolution. This allows us to separate spatial and temporal effects in the observed parameters.

STARE (Scandinavian Twin Auroral Radar Experiment): the ionospheric flow observations were made with a VHF radar system that consist of two radars operating near $140 \mathrm{MHz}$. The system observes in a stereoscopic manner phase velocities of plasma waves excited by electrons streaming through the ions in the ionospheric E-region. The measurements allow estimates of the total electron flow velocities over the field of view in northern Scandinavia. The STARE drift velocities have been derived as outlined in Nielsen and Schlegel $(1983,1985)$. The field of view of the radar system is $200000 \mathrm{~km}^{2}$; the spatial resolution is $15 \mathrm{~km}$ along the line-of-sight, and about $40 \mathrm{~km}$ at middle ranges, which results in a mean spatial resolution of about $25 \times 25 \mathrm{~km}^{2}$; the temporal resolution is $20 \mathrm{~s}$ (Nielsen et al., 1999).

IRIS (Ionosphere Riometer Imaging System): the ionospheric absorption measurements were made with a $38.2 \mathrm{MHz}$ riometer system which measures with good spatial and temporal resolution over a field of view of $60000 \mathrm{~km}^{2}$. The IRIS field-of-view is contained within the STARE field-of-view. The imaging riometer is located in Kilpisjaervi at $20.8^{\circ} \mathrm{E}$ and $69.05^{\circ} \mathrm{N}$ (Browne et al., 1995; Honary et al., 1995).
Solar wind plasma and magnetic field measurements on board WIND were used to determine the conditions in interplanetary space.

\section{Observations}

On May 4, 1998, the WIND spacecraft was located near $1.38 \times 10^{6} \mathrm{~km}$ from the Earth towards the Sun. At 0227 UT the detectors of solar wind speed and of the interplanetary magnetic field (IMF) recorded the arrival of a shock (Fig. 1). Just prior to the shock the wind speed was $550 \mathrm{~km} / \mathrm{s}$ and during the following minutes it increased to $700 \mathrm{~km} / \mathrm{s}$. During the following hour it further gradually increased to $800 \mathrm{~km} / \mathrm{s}$. For a speed between 700 and $800 \mathrm{~km} / \mathrm{s}$ it takes about $30 \mathrm{~min}$ to propagate from WIND to the Earth. The shock, if propagating at this speed, should arrive at the Earth near 0257 UT. At 0227 UT the magnetic field magni-

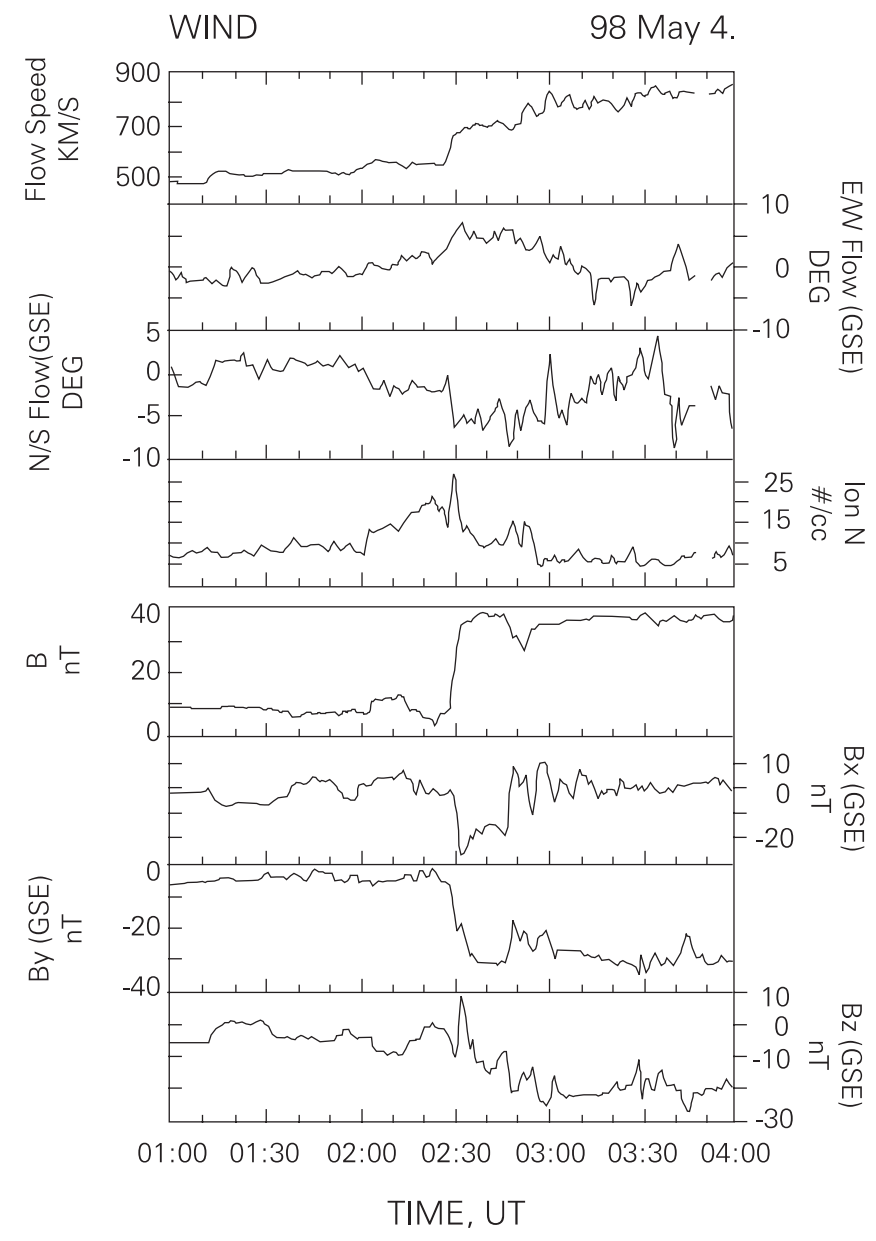

Fig. 1. WIND observations from top: solar wind bulk velocity; solar wind east-west component; solar wind north-south component; ion density; Interplanetary Magnetic Field (IMF) magnitude; IMF components. At 0227 UT a shock is detected as evidenced by the increase and rotation of the solar wind velocity and of the Interplanetary Magnetic Field (courtesy K. Ogilvie and R. Lepping at NASA/GSFC and CDAWeb). During the event he WIND spacecraft was located at a range of 216 Earth radii from the Earth in the direction towards the Sun 
tude at WIND also increased strongly, from $8 \mathrm{nT}$ before the shock to a value of $40 \mathrm{nT}$ after the shock. During 20 min following the shock onset the $B_{x}$ and $B_{y}$ components of the IMF decreased to $-25 \mathrm{nT}$, and $B_{z}$ decreased from a small negative value to $-30 \mathrm{nT}$. This corresponds to a change of polar angle of the IMF from $90^{\circ}$ to $140^{\circ}$. After $20 \mathrm{~min}$ the polar angle increased further to $160^{\circ}$, thus further increasing the coupling between the solar wind and the Earth system.

The magnetogram from Kakioka $\left(140.2^{\circ} \mathrm{E}, 36.2^{\circ} \mathrm{N}\right)$ shows in Fig. 2 the onset of the initial phase of the storm to be marked by a positive $H$ deviation at $0257 \mathrm{UT}$. The initial phase peaked at $0300 \mathrm{UT}$, and it lasted until 0304 UT, i.e. it had a duration of $7 \mathrm{~min}$. The $H$ component increased $50 \mathrm{nT}$ over the pre-event level. The onset time of 0257 UT is the projected time of arrival of the shock front at the Earth. At this Universal Time, $140^{\circ}$ eastern longitude is near local noon, i.e. near the sub-solar meridian. Assuming a cosine latitude variation of the ground magnetic disturbance, the disturbance at equator in the sub-solar region was near $60 \mathrm{nT}$.

For times near the Sudden Commencement Absorption, from 0250 to $0310 \mathrm{UT}$, Fig. 3a shows the latitudinal profile of convection flows observed with STARE superposed on the colour coded IRIS absorption measurements. Note that prior to the SCA at the latitudes where the absorption is most intense, i.e. between 69.0 and $69.6^{\circ}$, the flow speed is decreasing as the absorption is increasing. The absorption increased suddenly at 0257 UT to a value larger than $6 \mathrm{~dB}$ at all latitudes; the Magnetic Local Time is $2.5 \mathrm{~h}$ later; so the observations are near 0530 MLT, close to dawn. At the time of absorption onset, and for a short time after, the backscatter intensity decreased below threshold of the radar system so that no velocity could be determined in the high-absorption region. Backscatter was absent for about $2 \mathrm{~min}$, and returned then while the absorption was still high. Only at the lowest latitudes in the field of view could velocity vectors be determined during the whole time, and here they were reduced in magnitude. The particle precipitation covered IRIS field-of-view both in latitude and longitude.

Figure $3 \mathrm{~b}$ is a continuation in time of Fig. 3a. Several absorption regions are superimposed on a meandering pattern of ionospheric flows. This is especially clear in the latitude range from 68 to $70^{\circ} \mathrm{N}$ and from $0310 \mathrm{UT}$ until 0325 UT. The period of the meandering flow is about $330 \mathrm{~s}$. Note at $0322 \mathrm{UT}$ there is poleward and westward flow beginning to appear at high latitudes. Since the measurements were made near dawn, westward flows are identical with anti-sunward flows, characteristic of the polar cap. Whether the polar cap is associated with open or closed field lines depends on whether magnetic field line merging or friction is the dominating process driving the convection. In this sense the data indicate that the polar cap boundary is moving equatorward into the field-of-view. This pattern is further enhanced after 0330 UT.

Figure 4 shows IRIS absorption from 0300 UT to 0340 UT as a function of longitude and time. There are clearly recurrent intense absorption patches moving eastward (from the bottom to the top of the figure) across the field-of-view. The patch located near 0318 UT is drifting with a velocity of $\sim 2000 \mathrm{~m} / \mathrm{s}$, compatible with the estimated electron flow velocities at that time of $2200 \mathrm{~m} / \mathrm{s}$. The patches are drifting with a speed that is consistent with the $\mathbf{E} \times \mathbf{B}$ drift velocity.

The $X$-, and $Y$-components at ground based stations in the IMAGE magnetometer network are shown in Fig. 5 (Syrjaesuo et al., 1998). Following the SC there is a recovery of the $X$-component lasting a few minutes. This indicates a decrease in westward current intensity, coinciding with the time of loss of backscatter in the STARE system. This is also consistent with a decrease of the electron drift speed coinciding with the loss of backscatter. Following the SC the $Y$-component displays several peaks as is characteristic of Ps6 pulsations, which is the ground magnetic signature of Omega-bands (Kawasaki

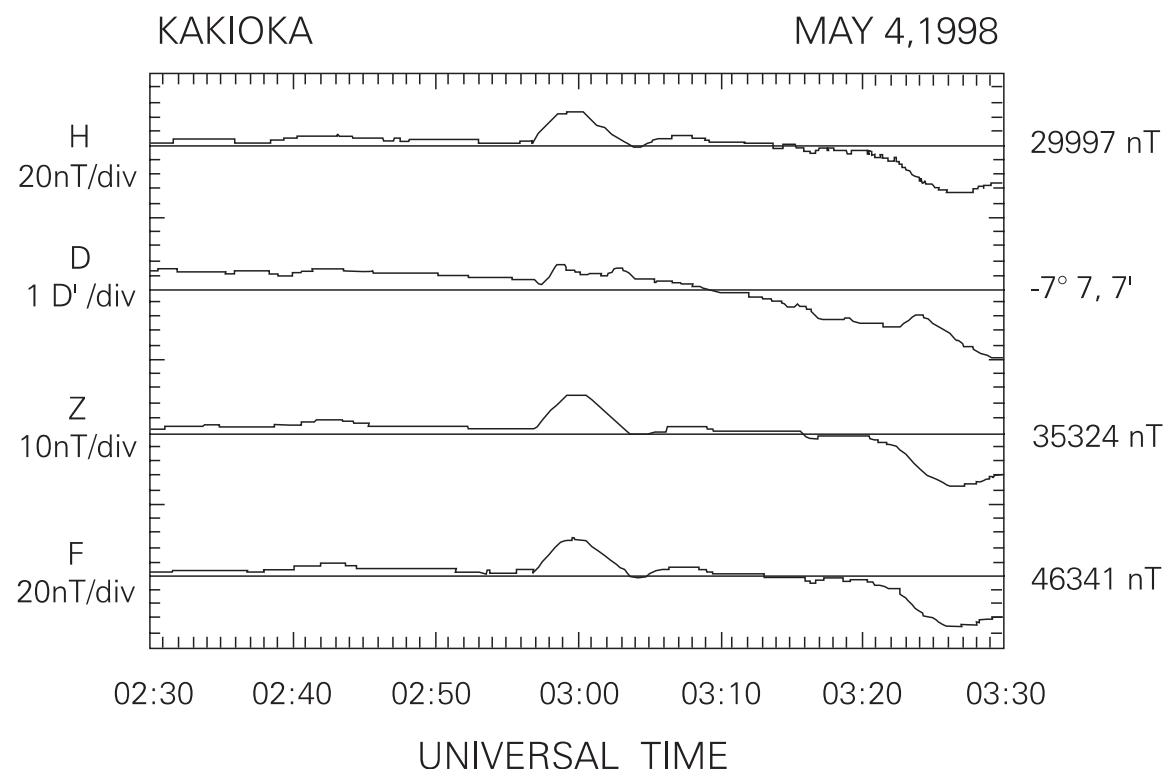

Fig. 2. Magnetogram from Kakioka, located at geographic latitude $36.2^{\circ} \mathrm{N}$ and longitude $140.2^{\circ} \mathrm{E}$. The Sudden Commencement of the storm is at 0257 UT. The initial phase of the storm lasts about $7 \mathrm{~min}$, and the decrease of the magnetic field in the main phase of the storm starts at 0320 UT (the data are supplied to WDC-C2, Kyoto from the observatory) 
STARE drift velocities and IRIS absorption;

1998. 5. 4

INT.TIME: $20 \mathrm{sec}$

SNR THRESHOLD: $1 \mathrm{~dB}$

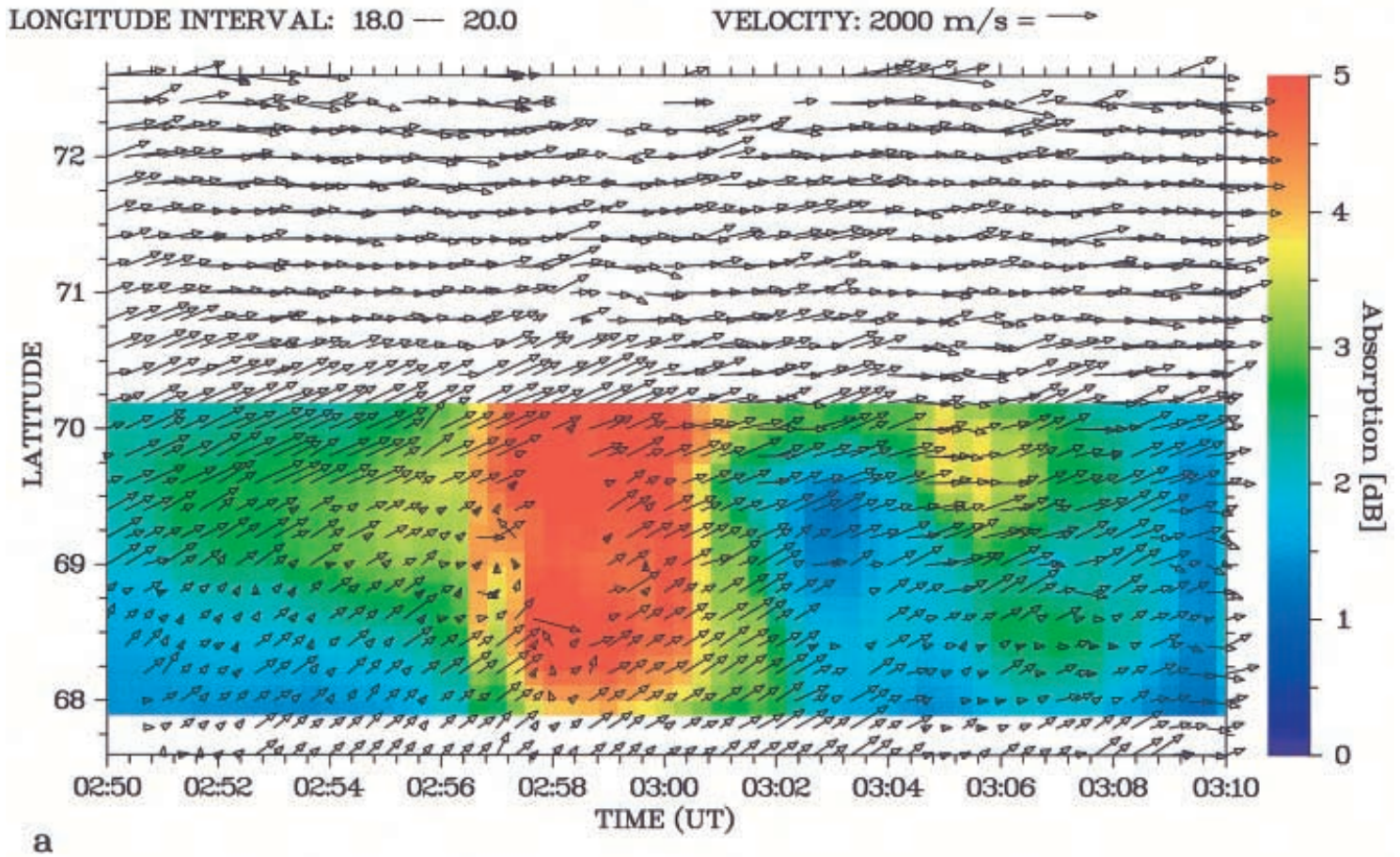

STARE drift velocities and IRIS absorption;

1998. 5. 4 INT.TIME: $20 \mathrm{sec}$ SNR THRESHOLD: $1 \mathrm{~dB}$

LONGITUDE INTERVAL: $18.0-20.0 \quad$ VELOCITY: $2000 \mathrm{~m} / \mathrm{s}=\longrightarrow$

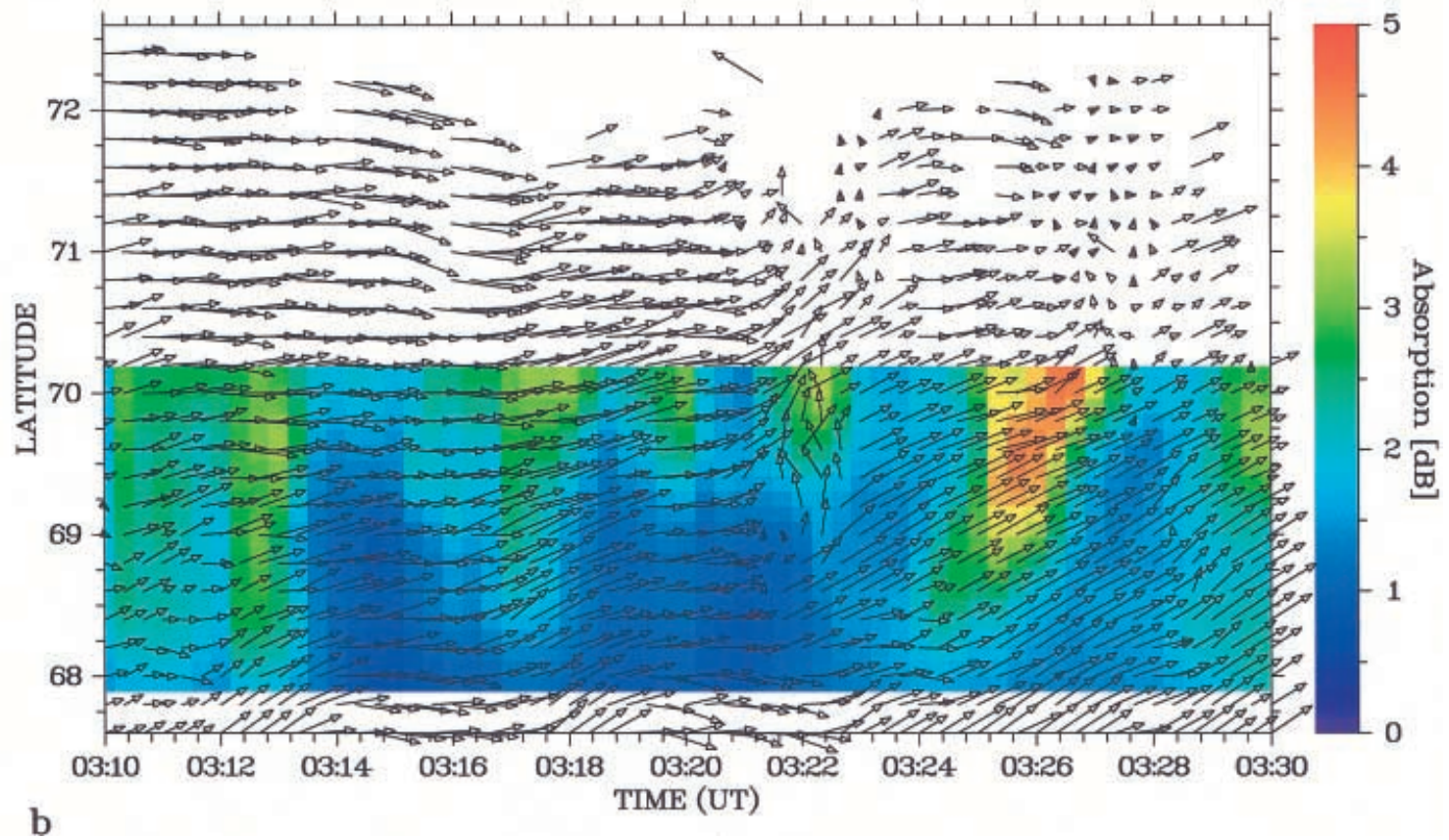

Fig. 3a, b. Latitude profile of STARE drift velocities and IRIS riometer absorption. At 0257 UT the Sudden Commencement Absorption is clearly to be seen. Especially after 0310 UT there are

and Rostoker, 1979; Gustaffson et al., 1981). Crosscorrelation of the $Y$-component measured at Andenes $\left(16.2^{\circ} \mathrm{E}\right)$ and Kevo $\left(27.0^{\circ} \mathrm{E}\right)$ yield a time delay at Kevo relative to Andenes of $170 \mathrm{~s}$, which corresponds to an clear meandering flows in the lower half of the latitude range. The absorption displays periodic increases following the SCA

eastward velocity of $2400 \mathrm{~m} / \mathrm{s}$, comparable with the other velocity estimates. The period of the pulsations is close to $300 \mathrm{~s}$; also this value is comparable with the period of the meandering flow obtained using STARE data. 


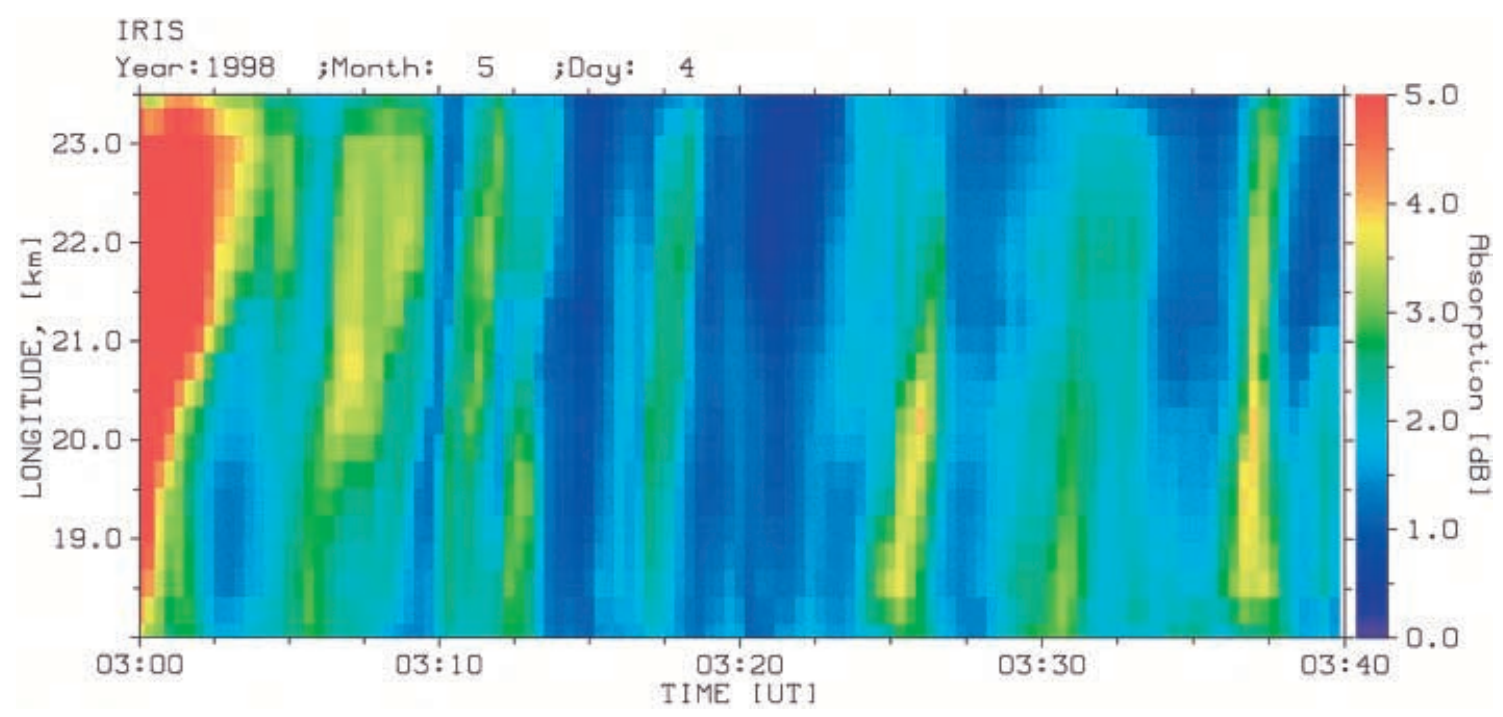

Fig. 4. Longitude profile of IRIS riometer absorption. Patches of absorption are repeatedly drifting across the field-of-view from west to east (from the bottom of the figure towards the top). The drift velocity is about $2000 \mathrm{~m} / \mathrm{s}$ comparable to the STARE drift velocities, indicating an $\mathbf{E} \times \mathbf{B}$ drift of the absorption patches

\section{Discussion}

First some effects of the shock on the geomagnetic cavity are outlined. It is followed by a discussion of the influence of the precipitation of energetic charged particles on the ionosphere/magnetosphere current circuit and on the electric fields in the ionosphere. Following the initial phase the observed effects of the shock on the electron flows in the ionosphere and on particle precipitation and its dynamics are discussed.

\subsection{The shock}

An interplanetary shock front arrived at the WIND spacecraft at 0227 UT, where a sudden increase in ion density, magnetic field strength, and solar wind velocity was observed. As the shocked solar wind arrived at the Earth it increased the pressure on the magnetosphere, and compressed the geomagnetic field. At the predicted arrival time at the Earth the magnetic field at equator of the sub-solar region increased by $60 \mathrm{nT}\left(\Delta B_{x}\right)$. This value of the ground magnetic field is consistent with the solar wind parameters. With reference to Parker (1967)

$\Delta B_{x} \sim \sqrt{P_{s}}$

and $P_{s} \sim \mathrm{NMV}^{2}$, where $\mathrm{N}=20 \mathrm{ion} / \mathrm{cm}^{3}, \mathrm{M}=1.7 \times$ $10^{-27} \mathrm{~kg}$, and $\mathrm{V}=700 \mathrm{~km} / \mathrm{s}$, the magnetic field change at equator in the sub-solar point is $40 \mathrm{nT}$, close enough for consistency with $60 \mathrm{nT}$ for the approximations involved.

When equilibrium with the shocked solar wind is reached, the distance to the sub-solar magnetopause is estimated to be

$\frac{\Delta B_{x}}{B_{0}} \sim 0.6\left(\frac{R_{E}}{R}\right)^{2}$ where $B_{0}=3.11 \times 10^{4} \mathrm{nT}$, and $R$ is the magnetopause distance in units of $R_{E}$, the Earth radius. In this approximation $R=6.8 R_{E}$; setting this value equal to McIlwain's parameter $L$, the corresponding magnetic latitude is 67.4 degrees. In northern Scandinavia geographic latitude is $2.5^{\circ}$ larger than geomagnetic latitude. Thus, if a steady state is achieved, the magnetopause is expected to map to a geographic latitude of about $70^{\circ}$ at local noon. Since the field of view of the STARE and IRIS experiments are close to dawn at the time of the event, the polar cap boundary in our field of view is expected to be at $70^{\circ}$ or at lower latitudes at dawn. This is consistent with the observations if the convection reversal observed with STARE after the SC is taken to coincide with the magnetopause.

The magnetic field strength at the magnetopause required to balance the pressure of the solar wind is estimated using,

$\frac{B^{2}}{2 \mu}=\frac{1}{2} N M V^{2}$

where $\mu=4 \pi \times 10^{-7}$. Using parameter values representative of conditions before and after the shock we find the magnetic field before and after the shock to be $57 \mathrm{nT}$ and $102 \mathrm{nT}$, respectively. Thus, the magnetic field in the magnetosphere increased by roughly a factor of 2 at the time of the shock.

In a dipole magnetic field the distance, $r$, in the equatorial plane to a magnetic field value of $B[\mathrm{nT}]$ is given by

$B=\frac{31100}{R^{3}}[\mathrm{nT}]$.

For $B_{1}=57 \mathrm{nT}, R_{1}=8.2 R_{E}$, and for $B_{2}=102 \mathrm{nT}$, $R_{2}=6.7 R_{E}$ not inconsistent with the previous estimates. 
IMAGE magnetometer network 1998-05-04

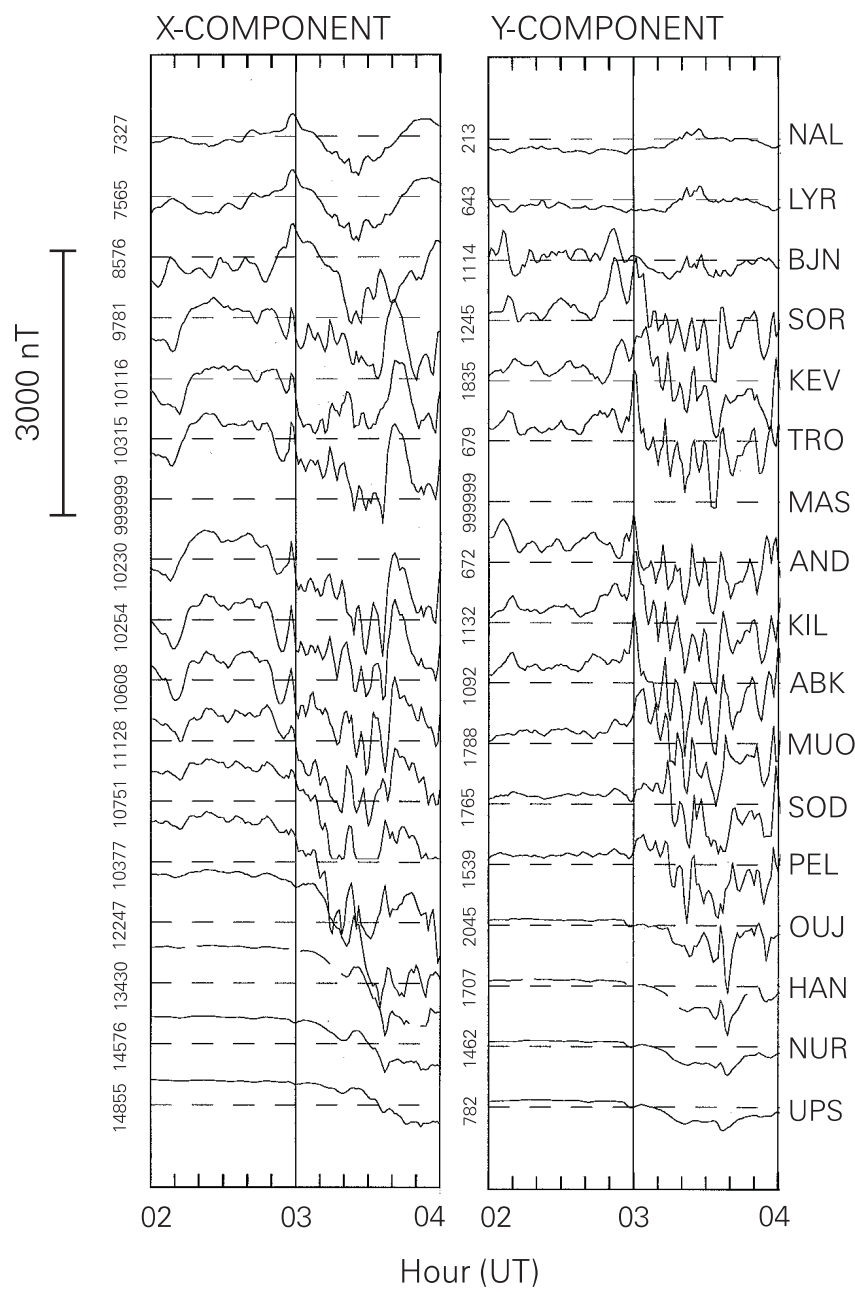

Fig. 5. $X$ - and $Y$-components of IMAGE magnetograms. The positive spike in the $X$-component just prior to $0300 \mathrm{UT}$ indicates the reduction of the ionospheric electric field magnitude associated with the rapidly increasing riometer absorption. The following series of positive spikes in the $Y$-component of especially MUO at the lowlatitude side of the STARE field of view, are consistent with Omega bands propagating overhead

\subsection{Particle precipitation}

At the time of the SCA there was a particle precipitation event in progress as evidenced by riometer absorption (Fig. 4). During the hours prior to arrival of the shock the absorption varied between 1 and $3 \mathrm{~dB}$. At $38.2 \mathrm{MHz}$ a $3 \mathrm{~dB}$ absorption corresponds (for example) to an electron flux of $F(E)=1.6 \times 10^{7}(6 / E)^{3} \mathrm{el} / \mathrm{cm}^{2}$ str $\mathrm{keV} \mathrm{s}$, where ' $E$ ' is the electron energy in $\mathrm{keV}$. Thus, an intense flux of electrons must have been present in the magnetosphere prior to the SC. Presumably strong pitch angle scattering caused electron fluxes of intensity up to a value of $F(E)$ to precipitate.

The maximum absorption during the SCA exceeded $6 \mathrm{~dB}$, i.e. more than a factor of 2 larger than the maxima prior to the shock. Since the absorption varies as the square root of the flux (all else remaining equal), there must have been at least a factor 4 increase in the flux precipitating during the SCA if the spectral shape remained constant (see e.g. Nielsen and Pomerantz, 1975; Nielsen et al. 1982). For constant spectral shape this flux increase would result if all electrons were accelerated to an energy increase of about $60 \%$.

The flux increase could be caused by acceleration of electrons of all energies by the same percentage (which would preserve the spectrum shape). This would be the case if the acceleration was caused by the gyro-cyclotron mechanism. The resulting energy, $T$, of a particle with initial energy, $T_{p a}$, parallel to the magnetic field, and perpendicular energy, $T_{p e}$, would then be (after Fillius and McIlwain, 1967)

$T=T_{p a}+T_{p e}\left(1+\frac{\Delta B}{B}\right)$

where $\Delta B$ is the magnetic field variation the electron experiences. Thus, the energy gain is pitch angle dependent. Since the value of $\Delta B$ was close to the value of $B$, the maximum energy change, which is for $90^{\circ}$ pitch angle, is a factor of two. Assuming strong pitch angle distribution, where electrons with large pitch angles are also quickly scattered into the loss cone, this may result in an effective mean energy increase of $60 \%$ as required to explain the observed increase in riometer absorption during the SCA. Since the increase in magnetic filed is thought to be large-scale, this interpretation would also account for the observed large-scale of the absorption, which starts simultaneously over the whole IRIS field of view.

\subsection{Ionospheric absorption and the Ionosphere/ magnetosphere current circuit}

During the initial phase of the storm the radar signal intensity was reduced. This may partly be caused by increased ionospheric absorption, as evident from the riometer measurements, but it may also be associated with dynamic changes in the ionospheric conductivity, which could cause a decrease of the ionospheric electric field, and so lead to a decrease of the radar signal intensity.

The radar signals propagate through the ionosphere at low elevation angles of between 11.0 and $4.8^{\circ}$ from horizontal. For a mean elevation angle of $7.9^{\circ}$, and taking into account that the ionosphere is spherical, this means that the absorption of the signal is 4.4 times larger than a signal propagating vertically through the ionosphere (as the riometer signal does). Taking into account the difference in frequency of the radar and riometer experiment, we find the radar signal should suffer an attenuation of $4.6 \mathrm{~dB}$ at the peak of the absorption during the initial phase. This may partly account for the loss of radar signal during the initial phase. It is however possible that rapid variations of the ionospheric conductivities also played a role.

The relationship between field aligned currents, ionospheric conductivities and electric fields have been studied by several authors, most recently by Davies and Lester (1999). Their results are based on statistical 
comparisons of simultaneously observed conductances and electric fields, and are representative of steady state conditions. The observed reduction of the electron drift speed while the absorption increased, during times prior to the $\mathrm{SC}$, is consistent with the findings of these authors. However, during the initial phase of the magnetic storm the particle precipitation was rising rapidly and became very intense before decreasing. The ionospheric radio wave absorption at $38.2 \mathrm{MHz}$ exceeded $6 \mathrm{~dB}$. Thus, this is an event with rapid time variations in the particle precipitation and therefore also rapid changes in the ionospheric conductivity, i.e. there is a situation far from steady state. The voltage driving the magnetosphere/ionosphere system changes at the time of the shock. However, it will take some time before this change has propagated to ground level. Our observations suggest that there are changes in the ionospheric electric field before the shock-induced voltage change has reached the ionosphere, namely, a sudden decreased of the electric field in response to the sudden increase in the Pedersen conductivity at the time of the SC. This can be interpreted to indicate that the current loop (ionospheric- and field aligned currents) contains an inductive component in the magnetospheric part of the current loop. The result suggest that dynamic variations in precipitation of energetic particles can have an influence on the magnitude of the ionospheric electric fields.

Since the SCA (which lasts only a few minutes during the initial phase of the storm, Fig. 3a) has the appearance of a large-scale event spatial gradients in the conductivities have been ignored in the following. Field aligned currents, $J_{\|}$, flowing along the geomagnetic field lines in the magnetosphere are closed in the ionosphere by the Pedersen current, $J_{p}$,

$J_{p}=\Sigma_{p} E$

where $\Sigma_{p}$ is the height integrated Pedersen conductivity and $E$ the horizontal ionospheric electric field. Here neutral winds have been ignored.

The field aligned currents are located where the divergence of the Pedersen current is non zero,

$J_{\|}=\nabla \times\left(\Sigma_{p} E\right)$

$J_{\|}=\Sigma_{p} \nabla \times E$

With a given distribution of field aligned currents, the closure currents in the ionosphere are only dependent on the (assumed homogeneous) conductivity and the spatial variations of the ionospheric electric field. If an increase of the conductivity occurred, but the field aligned currents were to stay constant, then the divergence of the electric field would have to decrease by the same amount. A decrease of the electric field divergence by, say, a factor 2, would be achieved if the field magnitude decreased by the same amount across space. This is the qualitative pattern of the observations during the time prior to the SC, where the riometer absorption slowly increases (the conductivity increases), and the electric field decreases with the tendency to keep the field aligned closure current in the ionosphere constant. While an increase in riometer absorption probably is associated with an increase in Pedersen conductivity, there is no general direct quantitative relationship. A derivation of field aligned currents based on riometer absortion values therefore does not seem sensible.

These equations and considerations are valid for steady state or slow variations in time. If there are rapid time variations the electrical properties of the magnetosphere/ionosphere current circuit have to be considered. For the purpose of discussing relative changes of the ionospheric conductivities, electric fields and currents, the electrical properties of the current loop are expressed in terms of electrical network elements (e.g. Bostroem, 1974). The ionosphere is equivalent to an Ohmic resistor $R$. The magnetosphere is simulated by an inductance $L$. $R$ and $L$ are in series and a current is driven through the elements by a voltage source, representing the solar wind magnetosphere interaction. Let the onset of the SCA be simulated by a sudden decrease of $R$ (onset of particle precipitation), see Fig. 6 . Owing to the inductance, the corresponding increase in current occurs slowly, with a time constant of $\tau=L / R$. Thus, during a time of order $\tau$, the voltage drop in the ionosphere, and therefore the ionospheric electric field, is reduced in magnitude. If the resistance is reduced a certain factor, then the electric field value is initially reduced by the same factor, and it then recovers exponentially. Therefore it is conceivable that for some time following the onset of the SCA the ionospheric electric fields are suppressed below the threshold value

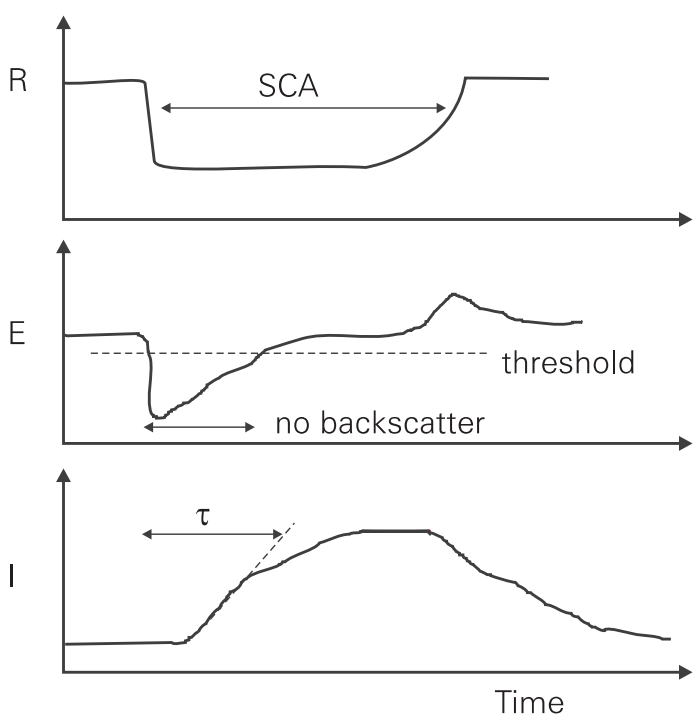

Fig. 6. Possible changes in the ionospheric electrical resistance $(R)$, electric field $(E)$ and current $(I)$ during a SCA. The sudden increase in particle precipitation reduces $R$; the current circuit tries to maintain the current and most of the voltage is placed over the magnetospheric inductance $(L)$ so that $E$ is reduced. With time constant, $\tau=L / R$, $E$ and $I$ approach the new equilibrium. While $E$ is less than the threshold (dashed line) for creating plasma instabilities there is no radar backscatter. For realistic values $R=0.1 \mathrm{Ohm}$ and $L=12$ Henry, $\tau=120 \mathrm{~s}$, which is consistent with the observations. Larger values of $\tau$ (i.e. larger values of $L$ and/or smaller values of $R$ ) would also be acceptable, since the observational restraint is only that $E$ is less than the threshold for about $120 \mathrm{~s}$ 
for production of ionospheric irregularities, which give rise to radar backscatter. It is the 'sluggishness' of the magnetospheric part of the current circuit that causes a reduction of the ionospheric electric fields below threshold for radar backscatter. Eventually, the ionospheric electric fields recovers and backscatter returns.

On the other hand when the resistance recovers the magnetospheric inductance will now cause the currents to initially stay large and then to slowly decrease. The voltage in the ionosphere will therefore be at increased values during recovery of the ionospheric resistance.

Thus, the electric fields are reduced in magnitude starting at the onset of the SCA and remaining for some time after (time duration of the order of $\tau$, or less), in agreement with the observations of loss of backscatter. The electric fields have recovered before the end of the SCA, and when the absorption decreases the fields remain large.

\subsection{Absorption patches}

One striking observation following the initial phase is the meandering flow in the ionosphere associated with patches of absorption. The flow pattern and the ground magnetometer data indicates that a Ps6 pulsation or an Omega band event is in progress.

Omega-bands are the dominant feature in the post midnight ionosphere during substorm recovery. Strong eastward plasma flows are superposed by auroral emissions with spatial forms of omegas strung along in the east-west direction. These forms are propagating eastward with the $\mathbf{E} \times \mathbf{B}$ electron drift velocity. The event has been interpreted as an eastward propagating string of alternating inward- and outward flowing field aligned currents. These field aligned currents are closed in the ionosphere by Petersen currents, driven by perturbation electric fields radiating outward (inward) from the intersection of the downward (upward) field aligned current with the ionosphere. These electric field perturbations results in circular electron flows in the ionosphere: clockwise directed around upward currents, and counter clockwise around downward currents. Superposition of these circular flows on the background electron flows causes the resulting total flow pattern to meander in latitude (Fig. 3b). It can easily be seen that when the flow meanders poleward and back again, then the plasma flows around the upward current. Figure $3 \mathrm{~b}$ shows that the regions equatorward of the poleward meandering flows are coinciding with regions of low absorption (dark blue absorption signature). Thus, the upward current regions are displaced from the regions of large riometer absorption, or large electron precipitation.

The ionosphere flows for the current event are meandering as in an Omega band event, and the ground-based magnetometer data showing Ps6 pulsations in the Y-component are consistent with that interpretation. Unfortunately, it was too late in the year for optical observations. In the framework of Omega bands the auroral emissions would be located in regions of upward current. Surprisingly, the expected regions of auroral emissions coincide with regions where the riometer absorption is very weak.

It has been proposed that Ps6 pulsations were caused by a region of enhanced electron precipitation drifting eastwards (Kawasaki and Rostoker, 1979). This scenario combined the magnetic pulsations with the auroral emissions, the eastward drift speed, and size of the Omega bands. Later Rostoker and Samson (1984) suggested that the Omega bands were excited by the Kelvin-Helmholtz instability on the boundary layer of the dawnside magnetotail plasma sheet. Pulkkinen et al. (1991) argued that the magnetospheric features connected to the Omega bands would have to be located at a distance from 6 to $13 R_{E}$ in the equatorial plane. Also Jorgensen et al. (1999) showed evidence to support the idea that omega bands map to the near Earth magnetosphere. The evidence for a near Earth source region lead to the suggestion that Omega bands are generated by the Kelvin-Helmholtz instability on the inner edge of the plasma sheet (Connors and Rostoker, 1993). The instability is excited if the relative bulk flow velocity in the plasma sheet is much higher than in the inner magnetosphere (provided other parameters such as the ratio of the mass densities on both sides of the inner edge is low).

Our evidence of Omega bands, as found in the meandering flows, is most pronounced in the lower part of the field of view, which is consistent with the source region being near the Earth. Also the observed speed of the plasma flows are high, of magnitude $2000 \mathrm{~m} / \mathrm{s}$, as is required by the theory. This evidence together with the observed meandering flows and magnetic pulsations, indicates that we are indeed observing an Omega band phenomena, or at least an event with similar characteristics. Omega bands are normally associated with the substorm recovery phase, when energy stored in the magnetotail is being released through the substorm onset, and transported back to the day side of the Earth. The intensification of the westward electrojet typical at such times, reflects the high plasma flow velocities in the dawn magnetosphere, which may lead to the Omega band formation. In the current event of a shock interaction with the Earth, there is an intensification of the convection velocities. Also in this case there is an enhanced transport of energy from the nightside to the dayside, and also here is a phenomena observed which is equivalent to an Omega band event. If the event was triggered by a substorm expansion starting at a time before the SC, then northern Scandinavia would have been in a negative bay at the time of the SC, but no negative bay was observed. The meandering flows are so closely associated with the SC that it is most likely triggered by the SC. It appears that generation of an east-west string of alternating inward/outward flowing field aligned currents drifting eastward with the background large $\mathbf{E} \times \mathbf{B}$ drift velocity, is a phenomena associated with rapid transport of large energies through the dawn side magnetosphere towards noon.

Hargreaves (1970) measured patches of riometer absorption to drift with speeds between $80 \mathrm{~m} / \mathrm{s}$ and 
$3300 \mathrm{~m} / \mathrm{s}$. The patches drifted eastward in the dawn ionosphere and westward in the dusk ionosphere, and since these are the general directions of the convection at dawn and dusk, it was suggested that the patches were drifting with the ionospheric plasma drift velocities. We have compared simultaneously measured drift velocities of absorption patches and ionospheric electron $\mathbf{E} \times \mathbf{B}$ drift velocities, and found agreement between these estimates. The energies of electrons dominating the riometer absorption are larger than $20 \mathrm{keV}$. At a radial distance of $6 R_{E}$ a gradient-curvature drifting $35 \mathrm{keV}$ electron needs about 120 min to drift around the Earth (Friedel et al., 1996). This drift period corresponds to a mean speed of the electrons of about $33 \mathrm{~km} / \mathrm{s}$. In a dipole field the corresponding speed in the high-latitude ionosphere would be $2 \mathrm{~km} / \mathrm{s}$. Higher (lower) energy electrons will move faster (slower). The speed of the $35 \mathrm{keV}$ electrons moving in the eastward direction in the morning magnetosphere is for the event we have examined compatible to the $\mathbf{E} \times \mathbf{B}$ speed. However, we see no reason why electrons of this energy should dominate the riometer absorption, and suggest that the relevant velocity with which to compare the drift velocity of the absorption patches is the $\mathbf{E} \times \mathbf{B}$ drift velocity.

\section{Summary}

Observations made during and after a shock interaction with the Earth have been shown to be consistent with ground magnetic measurements at local noon, with the magnetic field magnitude intensification in the magnetopause as evidenced by the increase in energetic particle precipitation, and with the expected compression of the magnetosphere as given by the radial distance to the magnetopause. A rapid and dynamic increase of the particle precipitation was suggested to be the cause of an observed short lasting reduction of the ionospheric electric field. Ionospheric electron drift velocities were found to meander in latitude revealing a pattern similar to the one known from Omega band events. Omega band events are normally associated with substorms, and thought to be triggered by the substorm expansion onset. In this case, however, the Omega band event was triggered by the SC. It thus appears that the Omega band event is a phenomena occurring together with rapid transport of large energies from the nightside to the dayside of the Earth. This evidence supports the idea that the Kelvin-Helmholtz instability is a mechanism for this type of event. Surprisingly, the regions in the ionosphere where the auroral luminosity is expected to be high (in the bright Omega band) we find the riometer absorption to be very low. The regions of high riometer absorption were found outside these regions. It was further shown that the absorption patches were drifting with the $\mathbf{E} \times \mathbf{B}$ drift velocity.

Acknowledgements. Plasma and interplanetary magnetic field data from the WIND spacecraft were obtained over the Web. We thank K. Ogilvie at NASA GSFC, R. Lepping at NASA GSFC, and
CDAWeb for making the data available. The STARE system is operated jointly by the Max Planck Institute for Aeronomie, Germany, and by the Finnish Meteorological Institute, Finland, in cooperation with SINTEF, University of Trondheim, Norway. The Imaging Riometer for Ionospheric Studies (IRIS) is funded by Particle Physics and Astronomy Research Council (PPARC) as one of the UK STP national facilities.

Topical Editor M. Lester thanks T. K. Yeoman and another referee for their help in evaluating this paper.

\section{References}

Akasofu, S.-I., Energy coupling between the solar wind and the magnetosphere, Space Sci. Rev., 28, 121-190, 1981.

Andre, D., and W. Baumjohan, Joint two-dimensional observations of ground magnetic and ionospheric electric fields associated with auroral zone currents. 5. Current systems associated with eastward drifting omega bands, J. Geophys., 50, 194-201, 1982.

Bostroem, R., Ionosphere-magnetosphere coupling, in Ed McCormac BM Magnetosphere physics, Reidel, Hingham, MA, 45-59, 1974.

Browne, S., J. K. Hargreaves, and B. Honary, An imaging riometer for ionospheric studies, Elect. Commun. Eng. J., 7(5), 209-217, 1995.

Connors, M., and G. Rostoker, Source mechanisms for morning auroral features, Geophys. Res. Lett., 20, 1535-1538, 1993.

Davies, J. A., and M. Lester, The relationship between electric fields, conductances and currents in the high latitude ionosphere: a statistical study using EISCAT data, Ann. Geophysicae, 17, 43-52, 1999.

Dungey, J. W., Interplanetary magnetic field and auroral zones, Phys. Res. Lett., 6, 47-48, 1961.

Fillius, R. W., and C. E. McIlwain, Adiabatic betatron acceleration by a geomagnetic storm, J. Geophys. Res., 72, 4011-4015, 1967.

Friedel, R. H. W., A. Korth, and G. Kremser, Substorm onsets observed by CRRES: determination of energetic particle source regions, J. Geophys. Res., 101, 13 137-13 154, 1996.

Gustaffson, G., W. Baumjohan, and I. Iversen, Multi-method observations and modelling of the three-dimensional currents associated with strong Ps6 event, J. Geophys. Res., 49, 138-154, 1981.

Hargreaves, J. K., Conjugate and closely-spaced observations of auroral radio absorption - IV. The movement of simple features, Planet. Sci., 18, 1691-1705, 1970.

Honary, F., S. Browne, and M. T. Riedveld, Observations of heaterinduced scintillation by an imaging riometer, in 7th International EISCAT Workshop, Corsica, 1995.

Jorgensen, A. M., H. E. Spence, T. J. Huges, and D. McDiarmid, A study of omega bands and Ps6 pulsations on the ground, at low altitudes and at geostationary orbit, J. Geophys. Res., 104, 14 705-14 715, 1999.

Kawasaki, K., and G. Rostoker, Perturbation magnetic fields and current systems associated with eastward drifting auroral structures, J. Geophys. Res., 84, 1464-1480, 1979.

Nielsen, E., and M. A. Pomerantz, Access of solar electrons to the polar regions, Planet. Space Sci., 23, 945-954, 1975.

Nielsen, E., and K. Schlegel, A first comparison of STARE and EISCAT electron drift velocity measurements, J. Geophys. Res., 88, 5745-5750, 1983.

Nielsen, E., and K. Schlegel, Coherent radar Doppler measurements and their relationship to the ionospheric electron drift velocity, J. Geophys. Res., 90, 3498-3504, 1985.

Nielsen, E., A. Korth, G. Kremser, and F. Marani, The electron pitch angle distribution at geosynchronous orbit associated with absorption spikes during the substorm expansion phase, J. Geophys. Res., 87, 887-894, 1982.

Nielsen, E., M. Bruns, I. Pardowitz, H. Perplies, L. Bemmann, P. Janhunen, and A. Huuskonen, STARE: 'observations of a field-aligned line current', Geophys. Res. Lett., 26, 1, 21-24, 1999. 
Opgenoorth, H. J., J. Oksman, K. U. Kaila, E. Nielsen, and W. Baumjohan, On the characteristics of eastward drifting omega bands in the morning sector of the auroral oval, J. Geophys. Res., 88, 9171-9185, 1983.

Ortner, J., B. Hultquist, R. R. Brown, T. R. Hartz, O. Holt, B. Landmark, J. L. Hook, and H. Leinbach, Cosmic noise absorption accompanying geomagnetic strom sudden commencements, J. Geophys. Res., 67, 4169-4186, 1962.

Parker, E. N., Disturbance of the geomagnetic field by the solar wind, in Physics of geomagnetic phenomena, Eds S. Matsushita and W. H. Campbell, vol II, 1152-1202, Academic Press, New York, 1967.

Pulkkinen, T. R., R. J. Pellinen, H. E. J. Koskinen, H. J. Opgenoorth, J. S. Murphree, V. Petrov, A. Zaitzev, and E. Friss-Christensen, Auroral signatures of substorm recovery phase: A case study, in Magnetospheric Substorms, Geophys, Monogr. Ser., vol 64, Ed J. R. Kan, T. A. Potemra, S. Kokubun, and T. Iijima, pp 333-341, AGU, Washington, D.C., 1991.

Rostoker, G., and J. C. Samson, Can substorm expansive phase effects and low frequency Pc magnetic pulsations be attributed to the same source mechanism?, Geophys. Res. Lett., 11, 271274, 1984.

Syrjaesuo, M. T., T. I. Pulkkinen, P. Janhunen, A. Viljanen, R. J. Pellinen, K. Kauristie, H. Opgenoorth, S. Wallman, P. Eglitis, P. Karlson, O. Amm, E. Nielsen, and C. Thomas, Observations of substrom electrodynamics using the MIRACLE network, Proc the International Conference on substorms4, Eds S. Kokubun and Y. Kamide, Astrophysics and Space Library, vol 238, Tarra Scientific Publishing Company/Kluwer Academic Publishers, 111-114, 1998. 